\title{
MANUALES EN BUENOS AIRES (1967-1987) EN LA BÚSQUEDA DE UNA "VULGATA ESCOLAR": RACCONTO DE UN PROCESO DE INICIACIÓN A LA INVESTIGACIÓN
}

Alejandra Deriard ${ }^{1}$

\section{RESUMEN}

En este escrito se muestran resultados de pesquisa de una experiencia de iniciación a la investigación de un grupo de futuros profesores de Matemática, en la búsqueda de regularidades en manuales escolares de escuelas primarias de Buenos Aires (1967-1987). Las conclusiones del trabajo indican que durante el período mencionado se ha vehiculizado, mediante los manuales, una "vulgata escolar", con una singular inclusión de las temáticas referidas a la teoría de conjuntos, mediante la cual se inició a los niños en el sistema de numeración y en el estudio de las operaciones aritméticas.

Palabras clave: manual, vulgata, primaria, matemática.

${ }^{1}$ Universidad Nacional de Tres de Febrero (UNTREF), Caseros, Argentina. 


\title{
MANUAIS EM BUENOS AIRES (1967-1987) NA \\ BUSCA DE UMA "VULGATA ESCOLAR": UMA \\ NARRATIVA DE UM PROCESSO DE INICIAÇÃO EM PESQUISA
}

\section{RESUMO}

Este artigo mostra os resultados de uma experiência de iniciação à pesquisa de um grupo de futuros professores de Matemática, na busca de regularidades nos manuais escolares das escolas primárias de Buenos Aires (1967-1987). As conclusões do trabalho indicam que, durante o período mencionado, foi transmitida através dos manuais uma "vulgata escolar", com uma inclusão única dos tópicos relacionados à teoria dos conjuntos, através dos quais as crianças foram iniciadas no sistema de numeração e no estudo de operações aritméticas.

Palavras-chave: manual, vulgata, primário, matemática.

\section{MANUALS IN BUENOS AIRES (1967-1987) IN THE SEARCH FOR A "SCHOOL VULGATE": A NARRATIVE OF A RESEARCH INITIATION PROCESS}

\begin{abstract}
This paper shows research results of an experience of initiation to the research of a group of future teachers of mathematics, in the search for regularities in school manuals of primary schools of Buenos Aires (1967-1987). The conclusions of the work indicate that during the aforementioned period a "school vulgate" has been vehiculized through the manuals, with a unique inclusion of the topics related to set theory, through which children were initiated in the numbering system and in the study of arithmetic operations.
\end{abstract}

Keywords: manuals, vulgate, primary, math.

\section{MANUELS À BUENOS AIRES (1967-1987) À LA RECHERCHE D'UNE "VULGATE SCOLAIRE": RACCONTO D'UN PROCESSUS D'OUVERTURE À L'ENQUÊTE}

\section{RÉSUMÉ}

Cet article présente les résultats d'une expérience d'initiation à la recherche d'un groupe de futurs professeurs de mathématiques, à la recherche de régularités dans les manuels scolaires des écoles primaires de Buenos Aires (1967-1987). Les conclusions du travail indiquent qu'au cours de la période susmentionnée, une "vulgate scolaire" a été véhiculée à travers les manuels, avec une inclusion unique des sujets liés à la théorie des ensembles, à travers lesquels les enfants ont été initiés dans le système de numérotation et dans l'étude des opérations arithmétiques.

Mots-clés: manuels, vulgate, primaire, maths. 


\section{INTRODUCCIÓN}

En este artículo se mostrarán los resultados parciales de una investigación aún en curso y los pasos llevados a cabo para su concreción. Esta pesquisa surge a raíz de una experiencia pedagógica de iniciación a la investigación en Historia de la Enseñanza de la Matemática llevada a cabo en un Instituto de Formación Docente y Técnica de la Provincia de Buenos Aires por 13 futuros profesores de Matemática en su último año de estudio (constituyéndose en investigadores amateurs), junto a su profesora de Metodología de Investigación en Educación Matemática ${ }^{2}$. Con el fin de pesquisar acerca de los contenidos de la enseñanza primaria en el período 19671987, en Buenos Aires, fue planteado el objetivo de analizar 30 manuales escolares del período 1967-1987 en Buenos Aires, con el fin de encontrar regularidades y diferencias entre ellos.

Es por ello que este artículo tiene el objetivo, por un lado, el de narrar la experiencia pedagógica de iniciación en la investigación en Historia de la Enseñanza de la Matemática de este grupo de investigadores; y por el otro, en el mismo entramado, el de ofrecer la elucidación de la "vulgata" (CHERVEL, 1991) (VALENTE, 2008) existente durante el período en cuestión, visibilizada inicialmente en los Documentos Curriculares oficiales de 1972 y 1981 (NACIÓN, 1972) (PASTORINO, 2009) y en las recomendaciones de la Conferencia Interamericana de Enseñanza de la Matemática (CIAEM), realizada en 1972 (RUIZ, 2011) (UNESCO, 1972) y plasmada en la casi totalidad de los manuales analizados.

\footnotetext{
2 Investigación "Los manuales escolares en el período 1967-1987, en Buenos Aires. La consolidación de una vulgata escolar", llevada a cabo durante 2019 en el Instituto de Formación Docente y Técnica Bernardo Houssay, de Bernal, Buenos Aires, Argentina. Dirección de investigación: Licenciada y Doctoranda Alejandra Deriard. Investigadores alumnos: Cabral, Micaela; Galán, Rocío; Gómez, Mónica; Jones, Mariel; Malatesta, Vanina; Mendicini, Belén; Plates, Javier; Rodríguez, Ayelén; Rodríguez, María Belén; Roldán, Walter; Sabino, Miriam; Salguero, Valeria; Szmygiel, Lucía.
} 


\section{ESTADO DEL ARTE EN ARGENTINA Y CONSIDERACIONES METODOLÓGICAS}

En la búsqueda de las investigaciones que enriquezcan un estado del arte de la Historia de la Enseñanza de la Matemática en Argentina, se han encontrado muy pocos estudios a la fecha de escritura de este artículo. Luego de infructuosa búsqueda a lo largo de 4 años, pueden citarse los escritos de autoras argentinas, como Falsetti (FALSETTI, 2015) acerca del estudio del movimiento de matemática moderna en la escuela secundaria argentina, y Deriard (DERIARD, 2019) acerca del análisis de la colección Así aprendemos Matemática. También se han encontrado estudios que refieren brevemente a la historia de la enseñanza de la matemática en tesis doctorales cuya temática principal es la Historia de la Educación. Tal es el caso de las tesis doctorales de Gvirtz (1996) y Tosi (2012). Es por ello que este trabajo se considera original, porque no se han encontrado autores que se refieran a la historia de la enseñanza de la matemática específicamente, en especial de la escuela primaria, en el período 1967-1987.

En cuanto a la metodología de trabajo, por el hecho de ser un campo inexplorado en Argentina, se ha debido recurrir a aquellos autores que, desde la perspectiva general de la Historia de la Cultura Escolar y de las disciplinas escolares, como Julia (2001), Choppin (2002), Chartier (1992, 2007) y Chervel (1991), fueron ingresando a la bibliografía argentina en los últimos años, de modo de hacer posible conocer los conceptos construidos por este campo de conocimiento, mediante sus escritos o mediante la lente de investigadores provenientes de países de Latinoamérica y de España, tales como Valente (2007, 2008) y Escolano Benito (2009), entre otros.

Es por medio de los conceptos de "representación", "apropiación" "cultura escolar", "manual escolar", y "vulgata escolar" y las relaciones entre éstos, que se ha podido organizar un relato historiográfico fundamentado en el estudio de los hechos histórico-educativos del período en cuestión (1967-1987). Los constructos mencionados fueron ampliados por el aporte a nuestro trabajo 
de la valiosa caja de herramientas del grupo Ghemat3. Sus escritos y su metodología de trabajo han sido una invalorable guía a la hora de analizar la totalidad de las fuentes primarias.

\section{CONTEXTO DE ESCRITURA DE LOS MANUALES ANALIZADOS}

Julia (2001) precisa el concepto de cultura escolar como la unión de dos conjuntos, el de las normas que delimitan los conocimientos a enseñar y las conductas a inculcar, por un lado, y el de las prácticas que permiten la transmisión de todos esos conocimientos y la incorporación de esos comportamientos, por el otro. Es, en lo referente a la historia de estas prácticas $\mathrm{y}$ a las normas que rigen el período educativo analizado, que este trabajo cobra sentido.

Los manuales encontrados en Buenos Aires y analizados (1967-1987) comprenden dos décadas entre las cuales se cuentan años de democracia y de dictadura militar en la Argentina, y entre las cuales la Reforma de la Matemática Moderna se instaura en las escuelas del nivel primario de la ciudad de Buenos Aires.

En 1969, año de edición del manual más antiguo de la serie analizada, la Capital Federal (que luego se llamaría Ciudad Autónoma de Buenos Aires), regía su educación primaria mediante el Programa de Educación Primaria de 1961 (NACION, 1961), elaborado por la Comisión Redactora del Proyecto de los Nuevos Programas para las Escuelas Primarias, cuya redacción se realizó durante el gobierno de Arturo Frondizi, quien fuera derrocado en abril de 1962.

En febrero de 1972 (RM 79/72), se publican los Lineamientos Curriculares de la Escuela Primaria. En este documento se observa, por primera

3 Grupo de Historia de la Enseñanza de la Matemática de Brasil, presidido por Wagner Rodrigues Valente. 
vez, la inclusión de temáticas referidas al Movimiento de Matemática Moderna:

\begin{abstract}
La enseñanza de la matemática en la escuela primaria determinará la actitud futura del alumno ante la materia. Si desde un principio se la enfoca como una actividad del alumno que lo llevará poco a poco a establecer relaciones, a pasar a expresarlas en un lenguaje simbólico, y de esta manera llegar gradualmente a la abstracción, no se crearan en el niño el temor ni la aversión hacia la materia, que es tan común observar en nuestro medio (NACIÓN, 1972, p. 35).
\end{abstract}

Dentro del mismo documento, podemos observar, como objetivo para el primer y el segundo año de la escuela primaria:

Estimular el desarrollo de la capacidad del alumnado para establecer relaciones [...] para que el niño: -se inicie en el lenguaje conjuntista; ordene y clasifique elementos; -se inicie en la idea de función; adquiera la noción de número natural a partir de la correspondencia entre conjuntos (NACIÓN, 1972, p.37)

En el año 1972 se realiza en Bahía Blanca, Argentina, la III CIAEM (Conferencia Interamericana de Enseñanza de la Matemática). En este importante evento, el matemático Luis Santaló, quien fuera designado en 1966 como agente de la reforma de la Matemática Moderna en América Latina, tuvo un protagonismo trascendental. En este acontecimiento se destaca el tratamiento de la Matemática Moderna en la Escuela Primaria, como uno de los 4 ejes prioritarios. Las recomendaciones de las anteriores conferencias (1961 y 1966), habían sido tomadas en cuenta por el Ministerio de Educación de la Nación Argentina, tal como se indica en las actas de la III CIAEM (RUIZ, 2011) (UNESCO, 1972). Dichas recomendaciones orientan hacia la introducción en la enseñanza de la matemática durante la escuela primaria acerca de la noción intuitiva de conjuntos, las relaciones de orden y equivalencia, la introducción de los números naturales y de las operaciones fundamentales desde sus propiedades estructurales (algebraicas) (UNESCO, 1972). 
En 1978, el gobierno nacional mediante la ley 21.810, transfiere los servicios educativos pre-primarios y primarios a la ciudad de Buenos Aires.

El 28 de noviembre de 1980, la Secretaría de Educación de la Municipalidad de Buenos Aires aprueba el Diseño Curricular que tiene vigencia a partir de la iniciación del curso lectivo de 1981 (PASTORINO, 2009). Este diseño curricular, en lo correspondiente a matemática, sugiere la implementación de la propuesta alrededor de cinco ejes: estructuras lógicomatemáticas, comunicación oral, psicomotricidad, organización espacial y organización temporal. Se evidencia en el mismo la presencia de los aportes teóricos de Piaget, Dienes, Revuz, Tapia, Castelnuovo, entre otros.

Será en 1987, luego de 4 años de gobierno democrático, que se pondrá en vigencia el Diseño Curricular para la Educación Primaria Común del Municipio de Buenos Aires, el que reemplazará definitivamente al Diseño Curricular de 1981. En esta norma curricular, ya no se manifiestan cuestiones referidas específicamente a la Reforma de la Matemática Moderna.

\section{PASOS EN LA BÚSQUEDA DE REGULARIDADES}

\section{PRIMER PASO: VISITA A LAS BIBLIOTECAS Y ENCUENTRO CON LAS FUENTES, UNA SUMA DE DIFICULTADES}

La idea de fuente adquiere su importancia fundamental si se repara en que todo conocimiento tiene siempre algo de exploración de "huellas". [...] Fuente histórica sería, en principio, todo aquel objeto material, instrumento o herramienta, símbolo o discurso intelectual, que procede de la creatividad humana, a cuyo través puede inferirse algo acerca de una determinada situación social en el tiempo. [...] Son la base empírica que sustenta un relato historiográfico (AROSTEGUI, 1995, p.378). 
La búsqueda de "huellas" en los manuales escolares del período 19671987, necesaria para esta investigación, resultó en una tarea complicada. Los alumnos investigadores se vieron en idénticos problemas que los investigadores expertos al momento de hallar las fuentes necesarias para dar respuesta a las preguntas iniciales, tendientes a la búsqueda de regularidades en los mismos. Una primera aproximación al objeto de investigación tuvo que ver con el aprendizaje del tratamiento de las fuentes históricas, en este caso, fuentes primarias.

Según Arostegui, las fuentes primarias son restos de la época en que ocurrió el evento (AROSTEGUI, 1995). Suponen registros dejados por aquella persona que experimentó o presenció un acontecimiento en cuestión, documentos originales, y se nos presentan de distinta manera, como por ejemplo en diarios, cartas, memorias, discursos, manuscritos, entrevistas, trabajos publicados o no publicados.

En la búsqueda de fuentes primarias del período a estudiar, se movilizó al equipo de investigación hacia la visita de 12 bibliotecas municipales, escolares y familiares; por alrededor de unas 130 horas reloj. Si bien el primer objetivo fue el encuentro de manuales de educación primaria, también se catalogó el resto de fuentes que podían ser de referencia a la hora del análisis.

Cabe destacar el celo y el asombro con que muchos de los 32 bibliotecarios y bibliotecarias, de las 12 bibliotecas municipales y escolares visitadas, recibieron al equipo y aportaron información valiosísima ante el pedido de manuales escolares que ya no se consultaban desde larga data. Una de las complicaciones encontradas, en la visita a las bibliotecas, fue que mucho material de esa época había sido desechado, o no estaba catalogado, ante lo cual, en algunos casos, los bibliotecarios permitían ingresar por detrás de bastidores a hurgar dentro de cajas cerradas, de libros amarillos, en mal estado, por cierto, pero valiosísimos para nosotros. Otra dificultad encontrada fue observar que muchos de los manuales no poseían autor. Al consultar con los bibliotecarios, indicaron que era una modalidad usual entre las editoriales de renombre no 
colocar los autores en los manuales que trataban más de una disciplina escolar, tal es el caso de algunos de los manuales analizados de editoriales como Estrada y Kapelusz.

Una mención especial corresponde realizar a los bibliotecarios de La Biblioteca y Hemeroteca del Maestro de la ciudad de Buenos Aires, quienes fueron de gran utilidad para el trabajo del equipo, brindando importante material con paciencia y dedicación. También fueron de vital importancia las bibliotecas familiares, destacando el recelo por parte de sus propietarios (35 personas consultadas) a la entrega del material. Todas estas bibliotecas nos dieron la posibilidad de encontrar algunos manuales en estado muy bueno, aunque en otros casos se nos hizo imposible la lectura por el efecto de la humedad y del paso del tiempo. El equipo, ante la necesidad de devolver casi inmediatamente el material consultado, debió aprender a digitalizar las fuentes mediante una app destinada a tal fin. Con todo el material digitalizado se formó un gran banco de datos que incluyó manuales escolares, revistas pedagógicas, diseños curriculares y actas de congresos correspondientes al período estudiado, ampliado por algún otro material que, si bien no estaba dentro del período histórico establecido, podía ser de ayuda para la comprensión del mismo, además de utilizado en otras investigaciones, como los manuales pertenecientes al período del escolanovismo, libros de pedagogía y textos destinados a la formación de maestros.

\section{SEGUNDO PASO: ESTUDIO DURANTE Y PARA EL ANÁLISIS DE LOS MANUALES}

A fin de profundizar aún más en la etapa a asimilar, fue necesario el estudio por parte del equipo de investigadores amateurs acerca de algunos temas de vital importancia a la hora del análisis de los materiales consultados, tales como: 
- Revisión y estudio sobre los textos de Piaget (CASTORINA, 1996) (PIAGET, 1978) y Dienes (DIENES, 1981) para la comprensión de la reforma de la Matemática Moderna;

- Estudio de la aproximación de la psicología genética y la matemática estructuralista, en el encuentro entre Piaget y Dieudonné, en el año 1952, llevando a Piaget a la conclusión de que en la totalidad de los estadíos de desarrollo de la inteligencia en los niños, los procesos de pensamiento se organizarían de forma estructurada, según las nociones definidas por los Bourbaki (ACZEL, 2009);

- Estudio de los antecedentes del Seminario de Royaumont, visibilización de las ideas acerca de las estructuras en la enseñanza de la matemática durante y a partir de la realización del Seminario en 1959, prescripción, en dicho evento, de las líneas centrales de la reforma de la matemática moderna (RUIZ, 2011);

- Estudio del modo en que la reforma comienza aplicándose en la enseñanza secundaria, y casi una década después, mediante el programa para el nivel primario, llevado a cabo por el International Study Group for Mathematics Learning - ISGML, conducido por Zoltán Dienes, se profundiza en la enseñanza primaria (VALENTE, 2012);

- Estudio del modo en que la reforma se orienta, mediante el Dr. Santaló, a Latinoamérica, tomando formato final en las Conferencias Interamericanas de Enseñanza de la Matemática, realizadas en los años 1961(Colombia), 1966 (Perú) y 1972 (Argentina) y eventos intermedios. (RUIZ, 2011);

- Estudio acerca de la tercera CIAEM (1972), realizada en Bahía Blanca. Evento en el cual, una de las 4 temáticas fundamentales fue la enseñanza de la matemática moderna en la escuela primaria y en la cual, autoridades del ministerio de educación de Argentina y de la 
OEA, manifiestan el interés por la reforma de la Matemática Moderna en nuestro país y Latinoamérica, dando cuenta de los dispositivos puestos en funcionamiento para la capacitación de los docentes. (UNESCO, 1972);

- Análisis de los diseños curriculares de 1972 y 1981 para la educación primaria, de la Nación y de la Municipalidad de Buenos Aires, respectivamente.

El análisis de otras fuentes, tales como documentos curriculares, diseños curriculares, revistas pedagógicas, actas de congresos y tesis doctorales, fue necesario para comprender el contexto de escritura de los manuales y para encontrar una relación entre ellas, además de darnos un indicio de cuál podría ser la vulgata que probablemente fuese vehiculizada en los textos escolares.

\title{
TERCER PASO: ANÁLISIS DE LOS MANUALES EN LA BÚSQUEDA DE REGULARIDADES, COMIENZO DE ELUCIDACIÓN DE LA "VULGATA" CONTENIDA EN ELLOS
}

\begin{abstract}
En cada época la enseñanza impartida por los maestros es idéntica, en líneas generales, en la misma disciplina y el mismo nivel. Todos o casi todos los manuales dicen lo mismo o prácticamente lo mismo. Los conceptos enseñados, la terminología utilizada, la ordenación de rúbricas y capítulos, la organización del cuerpo de conocimiento e incluso los ejemplos empleados o los tipos de ejercicios practicados, son idénticos, con escasas variantes. [...] La descripción y posterior análisis de esta vulgata constituyen la labor fundamental del historiador de una disciplina escolar (CHERVEL, 1991, p.90).
\end{abstract}

André Chervel, en su texto Historia de las Disciplinas Escolares (CHERVEL, 1991), destaca la importancia de utilizar los libros de texto como fuentes de investigación. En referencia a Chervel, Valente nos señala que en un cierto momento, para enseñar una materia, todos los libros de texto coinciden 
en lo que dicen, o casi; y es lo que, según Chervel, se llamó el fenómeno "vulgata" (VALENTE, 2008). En este sentido tomaremos el término "vulgata" para el análisis de los manuales.

La descripción y el análisis de la vulgata escolar es tarea fundamental del historiador de una disciplina escolar. Las vulgatas evolucionan o se transforman con el tiempo.

El manual escolar puede ser percibido como un espacio de representación de la memoria en que se ha materializado la cultura de la escuela, así como de las sensibilidades sociales de los agentes que lo producen y de los contextos que circulan (ESCOLANO BENITO, 2009).

Los manuales escolares se constituyen en un espacio de memoria por ser el soporte curricular a través del cual se vehicula el conocimiento academizado que las instituciones educativas han de transmitir, siendo, según Chartier, una "representación" del mundo que los produce y de la cultura que se los “apropia" (CHARTIER, 1992).

Los manuales escolares analizados en este estudio, se sitúan entre 1967 y 1987, período de auge del Movimiento de Matemática Moderna en Argentina. $\mathrm{El}$ análisis de estos libros hizo posible encontrar regularidades, coincidentes con nuestras observaciones durante el estudio de la normativa oficial directora de la currícula y en las recomendaciones de la CIAEM, a modo de anticipación de la vulgata escolar.

En la búsqueda de regularidades, observamos, adentrándonos en los capítulos de los manuales, similitudes en los conceptos y la terminología adoptada, así como en la organización de las secuencias de enseñanza, en el conjunto de ejemplos utilizados y en los ejercicios practicados. Es cierto que pudieron detectarse leves variaciones en algunos de los manuales, pero en líneas generales, las regularidades fueron muchas. Destacaremos en un apartado especial las diferencias encontradas en los manuales, que fueron mínimas.

En un segundo momento, como recorte del problema de investigación, 
se analizaron los capítulos de los libros que tratasen los temas de iniciación a la numeración y las cuatro operaciones. Del análisis fue posible inferir que la metodología de trabajo y los temas centrales se repetían, independientemente que fuesen de diferentes autores, ediciones, o editoriales, iniciando a los niños en matemática, en todos los casos, desde la lógica conjuntista. En el Cuadro 1 se expone un resumen de las unidades de análisis (orden de los temas, la terminología y simbología adoptada y la organización de las secuencias de enseñanza), correspondientes a los primeros seis años de la enseñanza primaria.

Cuadro 1- Resumen comparativo de unidades de análisis de Manuales durante el período 19671987 en la ciudad de Buenos Aires.

\begin{tabular}{|l|l|l|l|l|}
\hline & & & \\
\hline $\begin{array}{l}\text { Gregorio suma 1. } \\
\text { 1969 }\end{array}$ & Estrada & Idéntico & Idéntica & Muy similar \\
\hline $\begin{array}{l}\text { Matemática } \\
\text { goderna para 1er }\end{array}$ & Códex & Idéntico & Idéntica & Muy similar \\
\hline $\begin{array}{l}\text { Conjuntos, } \\
\text { números y } \\
\text { operaciones 1.1972 }\end{array}$ & ACME & Idéntico & Idéntica & Muy similar \\
\hline Matemática 1. 1984 & Susaeta & Idéntico & Idéntica & \begin{tabular}{l} 
Muy similar \\
\hline $\begin{array}{l}\text { Jugando con } \\
\text { Matemática 1. 1985 }\end{array}$
\end{tabular} \\
$\begin{array}{l}\text { Estrada } \\
\text { Matemática 1. 1985 }\end{array}$ & $\begin{array}{l}\text { Con algunas } \\
\text { variaciones. } \\
\text { Incluye } \\
\text { además otros } \\
\text { sistemas de } \\
\text { numeración. }\end{array}$ & Idéntica & $\begin{array}{l}\text { Las secuencias de } \\
\text { enseñanza fueron } \\
\text { modificadas } \\
\text { mínimamente, } \\
\text { incorporando grafía } \\
\text { con colores } \\
\text { variados. }\end{array}$ \\
\hline $\begin{array}{l}\text { Ruptura de } \\
\text { presentación }\end{array}$ & $\begin{array}{l}\text { Modificaciones } \\
\text { sustanciales. } \\
\text { Utiliza la noción }\end{array}$ & $\begin{array}{l}\text { Las secuencias de } \\
\text { enseñanza se } \\
\text { presentan a raíz de }\end{array}$ \\
\hline
\end{tabular}




\begin{tabular}{|c|c|c|c|c|}
\hline 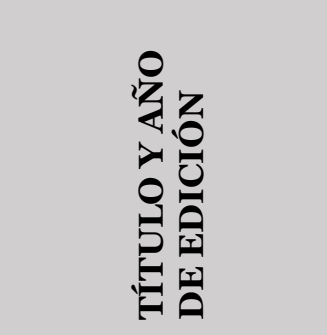 & 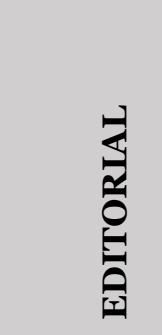 & 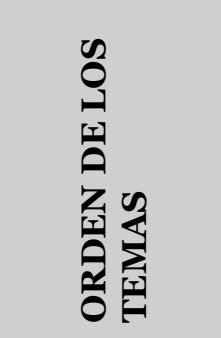 & 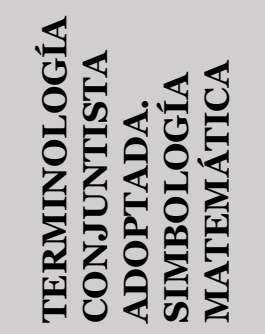 & 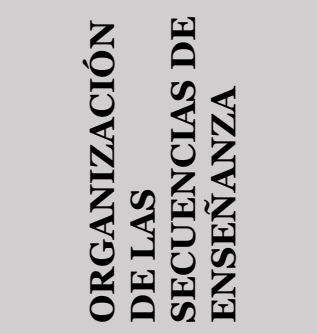 \\
\hline & & $\begin{array}{l}\text { de } \\
\text { contenidos. } \\
\text { Sin índice. }\end{array}$ & $\begin{array}{l}\text { de conjunto } \\
\text { pero no define } \\
\text { otros elementos } \\
\text { a partir de ella. }\end{array}$ & $\begin{array}{l}\text { una situación } \\
\text { problema, se } \\
\text { invierte la lógica del } \\
\text { paradigma anterior. } \\
\text { Se trabaja desde la } \\
\text { lógica Aplico, luego } \\
\text { Aprendo. No } \\
\text { existen definiciones } \\
\text { matemáticas } \\
\text { iniciales. }\end{array}$ \\
\hline Peldaño 2. 1969 & Kapelusz & Idéntico & Idéntica & Muy similar \\
\hline $\begin{array}{l}\text { Cuentos con } \\
\text { cuentas 2. } 1975\end{array}$ & Estrada & Idéntico & Idéntica & Muy similar \\
\hline $\begin{array}{l}\text { Nuevo Peldaño } 2 . \\
1976\end{array}$ & Kapelusz & Idéntico & Idéntica & Muy similar \\
\hline $\begin{array}{l}\text { Jugando con } \\
\text { Matemática 2. } 1981\end{array}$ & Estrada & $\begin{array}{l}\text { Con algunas } \\
\text { variaciones } \\
\text { sutiles. } \\
\text { Incluye } \\
\text { sistemas de } \\
\text { numeración. }\end{array}$ & Idéntica & $\begin{array}{l}\text { Las secuencias de } \\
\text { enseñanza se } \\
\text { modificaron } \\
\text { mínimamente, } \\
\text { incorporando grafía } \\
\text { con colores } \\
\text { variados. }\end{array}$ \\
\hline $\begin{array}{l}\text { Así Aprendemos } \\
\text { Matemática 2. } 1985\end{array}$ & Hachette & $\begin{array}{l}\text { Ruptura de } \\
\text { la lógica de } \\
\text { presentación } \\
\text { de } \\
\text { contenidos. } \\
\text { Sin índice. }\end{array}$ & $\begin{array}{l}\text { Modificaciones } \\
\text { sustanciales. } \\
\text { Utiliza la noción } \\
\text { de conjuntos } \\
\text { pero no define a } \\
\text { partir de ella. }\end{array}$ & $\begin{array}{l}\text { Las secuencias de } \\
\text { enseñanza se } \\
\text { presentan a raíz de } \\
\text { una situación } \\
\text { problema, se } \\
\text { invierte la lógica de } \\
\text { enseñanza. Se } \\
\text { trabaja desde la } \\
\text { lógica Aplico, luego } \\
\text { Aprendo. }\end{array}$ \\
\hline Matemática 3. 1971 & Teide & Idéntico & Idéntica & Muy similar \\
\hline Peldaño 3.1976 & Kapelusz & Idéntico & Idéntica & Muy similar \\
\hline
\end{tabular}




\begin{tabular}{|c|c|c|c|c|}
\hline 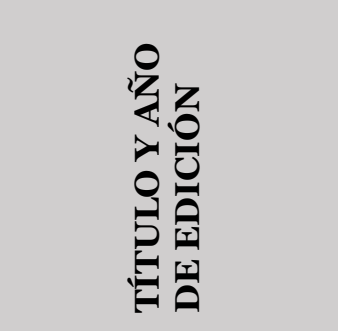 & 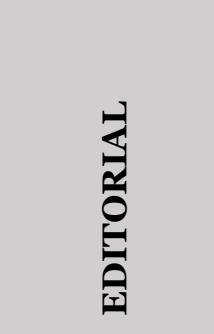 & 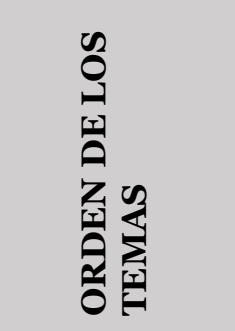 & 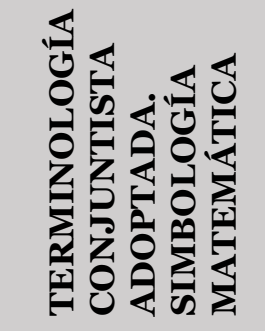 & 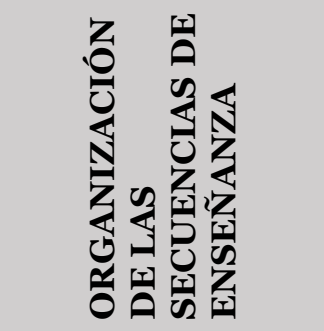 \\
\hline $\begin{array}{l}\text { Cuentos con } \\
\text { cuentas 3. } 1978\end{array}$ & Estrada & Idéntico & Idéntica & Muy similar \\
\hline $\begin{array}{l}\text { Jugando con } \\
\text { Matemática 3. } 1981\end{array}$ & Estrada & $\begin{array}{l}\text { Con algunas } \\
\text { variaciones } \\
\text { sutiles. } \\
\text { Incluye } \\
\text { sistemas de } \\
\text { numeración. }\end{array}$ & Idéntica & $\begin{array}{l}\text { Las secuencias de } \\
\text { enseñanza se } \\
\text { modificaron } \\
\text { mínimamente } \\
\text { incorporando grafía } \\
\text { con colores } \\
\text { variados. }\end{array}$ \\
\hline $\begin{array}{l}\text { El Mundo de los } \\
\text { Conjuntos } 4.1971\end{array}$ & $\begin{array}{l}\text { Magisterio } \\
\text { del Rio de la } \\
\text { Plata }\end{array}$ & Idéntico & Idéntica & Muy similar \\
\hline $\begin{array}{l}\text { Manual del Alumno } \\
\text { Bonaerense 4. } 1977\end{array}$ & Kapelusz & Idéntico & Idéntica & Muy similar \\
\hline $\begin{array}{l}\text { Jugando con } \\
\text { Matemática 4. } 1978\end{array}$ & Estrada & $\begin{array}{l}\text { Con algunas } \\
\text { variaciones } \\
\text { sutiles. } \\
\text { Incluye otros } \\
\text { sistemas de } \\
\text { numeración. }\end{array}$ & Idéntica & $\begin{array}{l}\text { Las secuencias de } \\
\text { enseñanza se } \\
\text { modificaron } \\
\text { mínimamente, } \\
\text { incorporando grafía } \\
\text { con colores } \\
\text { variados. }\end{array}$ \\
\hline $\begin{array}{l}\text { Aprendizaje y } \\
\text { Matemática 4. } 1981\end{array}$ & $\begin{array}{l}\text { Editorial } \\
\text { Plus Ultra }\end{array}$ & $\begin{array}{l}\text { Con } \\
\text { orientaciones } \\
\text { a los } \\
\text { maestros, } \\
\text { pero con la } \\
\text { misma lógica } \\
\text { de } \\
\text { presentación. }\end{array}$ & Idéntica & Muy similar \\
\hline $\begin{array}{l}\text { Manual del alumno } \\
\text { Bonaerense } 4.1982\end{array}$ & Kapelusz & Idéntico & Idéntica & Muy similar \\
\hline $\begin{array}{l}\text { Manual Bonaerense } \\
\text { Estrada } 4 \cdot 1984\end{array}$ & Estrada & Idéntico & Idéntica & Muy similar \\
\hline $\begin{array}{l}\text { Manual Kapelusz } \\
\text { Bonaerense } 4.1984\end{array}$ & Kapelusz & Idéntico & Idéntica & Muy similar \\
\hline
\end{tabular}




\begin{tabular}{|c|c|c|c|c|}
\hline 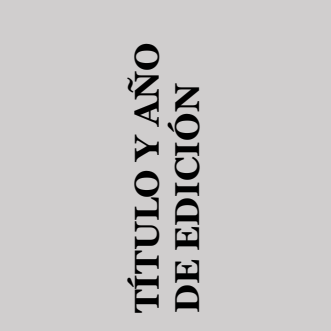 & 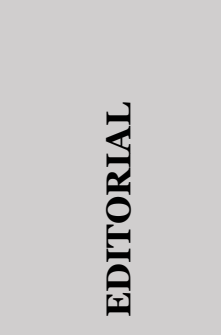 & 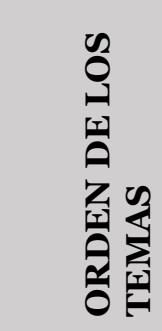 & 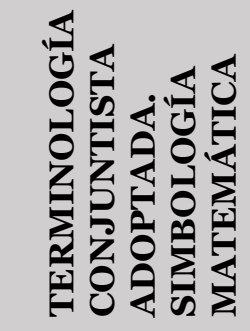 & 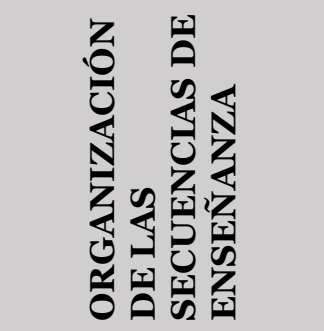 \\
\hline $\begin{array}{l}\text { Manual Bonaerense } \\
\text { 4. } 1986\end{array}$ & Santillana & Idéntico & Idéntica & Muy similar \\
\hline $\begin{array}{l}\text { Manual Estrada } \\
\text { Bonaerense } 4.1987\end{array}$ & Estrada & Idéntico & Idéntica & Muy similar \\
\hline $\begin{array}{l}\text { Manual Bonaerense } \\
\text { Estrada 5. } 1984\end{array}$ & Estrada & Idéntico & Idéntica & Muy similar \\
\hline $\begin{array}{l}\text { Manual Kapelusz } \\
\text { Bonaerense 5. } 1985\end{array}$ & Kapelusz & Idéntico & Idéntica & Muy similar \\
\hline $\begin{array}{l}\text { Manual Estrada } \\
\text { Bonaerense 5. } 1987\end{array}$ & Estrada & Idéntico & Idéntica & Muy similar \\
\hline $\begin{array}{l}\text { Manual Bonaerense } \\
5.1987\end{array}$ & Santillana & Idéntico & Idéntica & Muy similar \\
\hline $\begin{array}{l}\text { Aprendex } \\
\text { Matemática } 6.1984\end{array}$ & Estrada & Idéntico & Idéntica & $\begin{array}{l}\text { Los problemas } \\
\text { presentados son de } \\
\text { mayor complejidad. }\end{array}$ \\
\hline Matemática 6. 1987 & Hyspamérica & Idéntico & Idéntica & Muy similar \\
\hline
\end{tabular}

Fuente: Investigación "Los manuales escolares en el período 1967-1987, en Buenos Aires. La consolidación de una vulgata escolar", 2019. Instituto de Formación Docente y Técnica

Bernardo Houssay, Bernal, Buenos Aires, Argentina. Dirección de investigación: Licenciada y Doctoranda Alejandra Deriard. Investigadores alumnos: Cabral, Micaela; Galán, Rocío; Gómez, Mónica; Jones, Mariel; Malatesta, Vanina; Mendicini, Belén; Plates, Javier; Rodríguez, Ayelén; Rodríguez, María Belén; Roldán, Walter; Sabino, Miriam; Salguero, Valeria; Szmygiel, Lucía.

En casi todos los manuales observados, se reitera la lógica de presentación de los contenidos y el tratamiento similar de los temas, según esta secuencia: Al inicio, se presenta la noción de conjunto y elementos ${ }^{4}$, diagramas de Venn o Carroll, definición de conjunto vacío, conjunto unitario, pasando a la

\footnotetext{
4 Recordemos que solo pueden presentarse, mostrarse estas nociones, debido a que "conjunto", "elemento" y "pertenencia" son términos primitivos y no pueden definirse.
} 
iniciación en el sistema de numeración y de las operaciones elementales, siempre desde la noción de cardinal, de operaciones (unión, intersección y diferencia) y de correspondencia entre conjuntos.

Este formato de enseñanza del sistema de numeración decimal, se corresponde con lo que Valente señala, citando a Dienes:

La propuesta de un programa moderno para la enseñanza elemental tiene en cuenta cuatro caminos que deben seguirse paralela y progresivamente: el camino algebraico, el aritmético, el lógico y el geométrico. Para la enseñanza del sistema de numeración, por ejemplo, perseguir paralela y progresivamente significa, algebraicamente: iniciar la enseñanza con nociones conjuntistas, con diagramas de Venn o de Carroll, aritméticamente: trabajar el aprendizaje del número natural a partir de las nociones de conjunto; lógicamente: considerar las propiedades y atributos de los objetos o conjuntos de objetos; geométricamente, considerar las nociones de topología (VALENTE, 2012, p. 1430).

Esta regularidad se observa en los manuales, con excepción de dos de ellos de la editorial Hachette (Así Aprendemos Matemática), los que merecen un tratamiento especial. La particularidad observada en casi todos ellos, es la de la introducción del concepto de número, a partir del concepto de cardinal de un conjunto $^{5}$. Coincide lo observado a este respecto en los manuales, con los escritos de Dienes:

El número es una abstracción. Los números no tienen existencia real. Los números son propiedades, pero se trata de propiedades relativas a conjuntos de objetos, no a los objetos mismos. [...] Por esto existe un mundo intermedio entre el de los objetos y el de los números, a saber, el mundo de los conjuntos (DIENES, 1981, p. 15).

Recordemos que el Diseño Curricular de la Escuela Primaria de 1972

${ }^{5}$ Se denomina cardinal de un conjunto a la cantidad de elementos que el conjunto posee. 
indicaba:

\begin{abstract}
Si desde un principio se la enfoca como una actividad del alumno que lo llevará poco a poco a establecer relaciones, a pasar a expresarlas en un lenguaje simbólico, y de esta manera llegar gradualmente a la abstracción. [...] Lográndose que se inicie en el lenguaje conjuntista; -ordene y clasifique elementos; -se inicie en la idea de función; -adquiera la noción de número natural a partir de la correspondencia entre conjuntos (NACIÓN, 1972, p. 37).
\end{abstract}

De la lectura de los manuales y del conocimiento del contexto de producción de los mismos, podemos confirmar los dichos de Valente:

Como resultado del contexto, la organización curricular y la enseñanza de la matemática para niños sufre una transformación radical: el número no podrá más ser enseñado al inicio de la escolaridad. El número es un elemento abstracto, el cual debe quedar lejos de los niños [...] Número es propiedad. Y en este caso, se altera el orden vigente hace siglos (VALENTE, 2012, p.1436).

Es entonces que, según esta alteración del orden, manifestado por Valente, los primeros contactos de los niños con la matemática en los primeros años de escolaridad primaria, en Buenos Aires, se lograría a través del Algebra, las relaciones y solo en último término, los números. En los manuales analizados queda plasmado que los primeros elementos del Algebra para niños se constituyeron en nuevos contenidos, tal como lo indicaban los Lineamientos Curriculares de 1972, el Diseño Curricular de 1981, y las recomendaciones de la CIAEM. Estos nuevos contenidos, avanzando con la terminología de Valente, serían los "nuevos elementares" (VALENTE, 2012) entendidos como nuevas formas fundamentales del conocimiento y constituirían una nueva matemática escolar, construida a partir de la Teoría de Conjuntos.

Será así que el lenguaje conjuntista se constituirá en parte fundamental de la vulgata escolar durante el auge de la Matemática Moderna en Buenos 
Aires, visibilizado en los manuales escolares, en los Diseños Curriculares Oficiales y en las recomendaciones de la III CIAEM.

Como excepción a estas conclusiones, podemos referirnos a los manuales editados por Hachette bajo la dirección de Elsa Bergadá Mugica, Así Aprendemos Matemática. Estos manuales escaparon a la lógica descripta anteriormente y fueron utilizados hasta el año 2002. Los mismos serán analizados en profundidad en una segunda parte de esta investigación, aún en proceso.

\section{ALGUNAS CONCLUSIONES HASTA EL MOMENTO}

Según Chervel (1991) la primera tarea del historiador de las disciplinas escolares es el estudio de los contenidos que componen la enseñanza de la disciplina, siendo ese estudio necesariamente encaminado y contextualizado por variadas fuentes. En el caso de este trabajo, las fuentes primordiales fueron 30 manuales escolares, aunque también se consultaron los documentos curriculares que llevaron adelante la reforma de la Matemática Moderna en Buenos Aires, y las recomendaciones de la III CIAEM (RUIZ, 2011) (UNESCO, 1972). Este entramado de fuentes, sumados al estudio de los acontecimientos históricos de la época, permitió conocer la "cultura escolar" del momento histórico estudiado (JULIA, 2001), facilitando el análisis de los 30 manuales del período seleccionado (solo en lo que se refiere a los contenidos de Teoría de conjuntos, numeración y operaciones).

Entendemos que la Historia de la Enseñanza de la Matemática, está fundada sobre las discontinuidades y rupturas. En el caso del Movimiento de la Matemática Moderna en Buenos Aires, se produjo una apropiación (CHARTIER, 1992), a modo de relación de dominación, de las recomendaciones de la III CIAEM para América Latina y de las prescripciones de los Diseños Curriculares oficiales, que aparejó una ruptura forzada con los formatos 
pedagógicos vigentes al momento de la reforma definiendo, a partir de allí, una "vulgata escolar" que, según se observó en los manuales, duraría al menos dos décadas.

Los resultados de esta investigación mostraron que en casi la totalidad de los manuales escolares referentes a la educación primaria analizados, se visibilizó esta nueva vulgata, presente en los diseños curriculares de la ciudad de Buenos Aires y en las recomendaciones del III CIAEM correspondientes a la enseñanza de los primeros años de escolaridad, en lo que se refiere al tratamiento de los temas desde las estructuras algebraicas, a la enseñanza del concepto de número y del sistema de numeración a partir de la Teoría de Conjuntos.

Es así como la Teoría de Conjuntos ocupó un lugar de privilegio en, al menos, los primero seis años de la enseñanza de la matemática de la escuela, en la ciudad de Buenos Aires, siendo utilizada como una herramienta para analizar, clasificar y ordenar los conocimientos a adquirir posteriormente, confeccionando una compleja estructura cognitiva que el alumno debía utilizar durante todo su paso por la escuela primaria.

En este artículo se mostró, además, la iniciación a la investigación de 13 futuros profesores de Matemática, en Historia de la Enseñanza de la Matemática. A ese respecto cabe destacar lo inédito de la experiencia en cuando al estudio de la Historia de la Enseñanza de la Matemática en Argentina y la importancia de los resultados observados. El equipo de trabajo comprendió la relevancia de la búsqueda, el cuidado y el análisis de las fuentes históricas, del trabajo cooperativo y del estudio constante antes y durante el proceso investigativo. Esta experiencia continuará con la profundización de la pesquisa durante 2020. 


\section{BIBLIOGRAFÍA}

ABAD, Diego. Manual Bonaerense 4. Buenos Aires: Santillana, 1986.

ABAD, Diego. Manual Bonaerense 5. Buenos Aires: Santillana, 1987.

ACZEL, Amir; JAWERBAUM, Silvia; BARBA, Julieta. El artista y el matemático: la historia de Nicolas Bourbaki, el genio matemático que nunca existió. Madrid: Gedisa, 2009.

ADAIME, Dora; BERGADÁ MUGICA, Elsa; GARCÍA CAMPRA, Haydée. Así aprendemos matemática 1. Buenos Aires: Hachette, 1985.

ADAIME, Dora; BERGADÁ MUGICA, Elsa; MUSANTE, Pilar. Así aprendemos matemática 2. Buenos Aires: Hachette, 1985.

ALVAREZ, María; REY, María; SAGGESE, Delia; TORELLI, Alicia. Aprendizaje y matemática 4. Buenos Aires: Plus Ultra, 1981.

ARÓSTEGUI, Julio. La investigación histórica: teoría y método. Barcelona: Crítica, 1995.

ASTOLFI, Juan; CHAN, Luis; FESQUET, Josefina. Manual del alumno Bonaerense 4. Buenos Aires: Kapelusz, 1982.

BARGIELA, Heriberto; PIZZO, Aldo; REYNA, Jorge. Matemática 6. Buenos Aires: Hyspamérica, 1987.

CASTORINA, José. El legado de Piaget para la educación. El desafío. Revista del Instituto de Investigaciones de la Facultad de Psicología, v. 2, n. 3 , p. 21-35, 1996.

CHARTIER, Roger. El mundo como representación. Barcelona: Gedisa, 1992.

CHARTIER, Roger. La historia o la lectura del tiempo. Barcelona: Gedisa, 2007.

CHERVEL, André. Historia de las disciplinas escolares: reflexiones sobre un campo de investigación. Revista de Educación, n. 295, p. 59-111, 1991.

CHOPPIN, Alain. O historiador e o livro escolar. Revista História da Educação, v. 6, n. 11, p. 5-24, jan./jun. 2002.

D'AMELIO DE SCHEFINI, Nélida; SCHEFINI, Alberto. Cuentos con cuentas: Matemática moderna para $3^{\circ}$ grado. Buenos Aires: Estrada, 1978. 
D'AMELIO DE SCHEFINI, Nélida; SCHEFINI, Alberto; BESIO, Alfredo.

Cuentos con cuentas: Matemática moderna para $2^{\circ}$ grado. Buenos Aires:

Estrada, 1975 .

DAVILA, Edgardo; GABBA, Pablo; RATTO, Jorge. Matemática Moderna para primer grado. Buenos Aires: Codex, 1969.

DERIARD, Alejandra. Los libros del maestro Así aprendemos matemática: un cambio de paradigma en la enseñanza de la matemática, en los 80 , en la ciudad de Buenos Aires. Revista de História da Educação Matemática, v. 5, n. 1, 2019 .

DESCHAMPS, María. Matemática 3. Barcelona: Teide, 1971.

DIENES, Zoltan Paul. La Matemática moderna en la enseñanza primaria. Barcelona: Teide, 1981.

DISEÑO CURRICULAR CIUDAD DE BUENOS AIRES, Decreto 07961987.

DOS REIS DE MAMONE, Perla; JÁUREGUI, Jovita. Conjuntos, números y operaciones. Primer grado, cuaderno 1. Buenos Aires: ACME, 1972.

ESCOLANO BENITO, Agustín. El manual escolar y la cultura profesional de los docentes. Tendencias pedagógicas, n. 14, p. 169-180, 2009.

FERRARI, Cristina. Gregorio suma. Texto para la enseñanza de la matemática moderna en el 1er grado. Buenos Aires: Estrda, 1969.

GALÁN, Elvira; SCAGLIA, Horacio. El mundo de los conjuntos, Cuarto

Grado. Buenos Aires: Magisterio del Rio de la Plata, 1971.

JULIA, Dominique. A cultura escolar como objeto histórico. Revista

Brasileira de História da Educação, v. 1, n. 1 [1], p. 9-43, 2012.

LEY 21810. Transferencia de servicios educativos. 1978.

LOPEZ, Jorge. Nuevo Peldaño 2. Matemática para la escuela moderna.

Buenos Aires: Kapelusz, 1976.

MANUAL Bonaerense Estrada 4. Buenos Aires: Estrada, 1987.

MANUAL Bonaerense Estrada 4. Buenos Aires: Estrada, 1984.

MANUAL Bonaerense Estrada 5. Buenos Aires: Estrada, 1985.

MANUAL del Alumno Bonaerense 4. Buenos Aires: Kapelusz, 1987.

MANUAL Estrada Bonaerense 5. Buenos Aires: Estrada, 1987. 
MANUAL Kapelusz Bonaerense 4. Buenos Aires: Kapelusz, 1984.

MANUAL Kapelusz Bonaerense 5. Buenos Aires: Kapelusz, 1985.

NACIÓN, M DE EDUCACIÓN. Aprobación de organización curricular.

Resolucion n ${ }^{0}$ 237. Buenos Aires, 1979.

NACIÓN, M. DE EDUCACIÓN. Lineamientos Curriculares para la Escuela Primaria. Argentina. 1972

NACIÓN, M. DE EDUCACIÓN. Plan de Estudios de Educacion Primaria.

Resolución del Consejo Nacional de educación. 1961.

OCHOA, Luis. Matemática 1. Medellín: Susaeta, 1984.

PASTORINO, Héctor Oscar. Una crónica histórica del sistema educativo de la ciudad de Buenos Aires 1978-1998. Buenos Aires: UBA, 2015.

PELDAÑO 2. Mi segundo cuaderno de cálculo. Buenos Aires: Kapelusz, 1969.

PELDAÑO 3. Matemática para la Escuela Moderna Bonaerense. Buenos Aires: Kapelusz, 1976.

PIAGET, Jean. La equilibración de las estructuras cognitivas: problema central del desarrollo. Madrid: Siglo XXI, 1978.

RUIZ, Angel. El CIAEM y las organizaciones internacionales de Educación Matemática en América Latina. Cuadernos de Investigación y Formación en Educación Matemática, p. 15-25, 2011.

TAPIA, Nelly; BIBILONI, Alicia. Aprendex Matemática 6. Buenos Aires: Estrada, 1984.

TAPIA, Nelly; BIBILONI, Alicia. Jugando con Matemática 3. Buenos Aires: Estrada, 1981.

TAPIA, Nelly; BIBILONI, Alicia. Jugando con Matemática 4. Buenos Aires: Estrada, 1978.

TAPIA, Nelly; BIBILONI, Alicia. Jugando con Matemática 2. Buenos Aires: Estrada, 1981.

TAPIA, Nelly; BIBILONI, Alicia. Jugando con Matemática 1. Buenos Aires: Estrada, 1985.

UNESCO. Informe de la tercera Conferencia Interamericana sobre Educación Matemática. Bahia Blanca, 1972. 
VALENTE, Wagner Rodrigues. História da Educação Matemática: interrogações metodológicas. Revemat - Revista Eletrônica de Educação Matemática, v. 2, n. 1, p. 28-49, 2007.

VALENTE, Wagner Rodrigues. Livro didático e educação matemática: uma história inseparável. Revista Zetetiké, v. 16, n. 30, p. 139-162, 2008.

VALENTE, Wagner Rodrigues. O que é número?: produção, circulação e apropriação da Matemática Moderna para crianças. Bolema - Boletim de Educação Matemática, v. 26, n. 44, p. 1417-1442, 2012.

TAPIA, Nelly; BIBILONI, Alicia. Aprendex Matemática 6. Buenos Aires: Estrada, 1984.

TAPIA, Nelly; BIBILONI, Alicia. Jugando con Matemática 3. Buenos Aires: Estrada, 1981.

TAPIA, Nelly; BIBILONI, Alicia. Jugando con Matemática 4. Buenos Aires: Estrada, 1978.

TAPIA, Nelly; BIBILONI, Alicia. Jugando con Matemática 2. Buenos Aires: Estrada, 1981.

TAPIA, Nelly; BIBILONI, Alicia. Jugando con Matemática 1. Buenos Aires: Estrada, 1985.

ALEJANDRA DERIARD é professora de matemática, especialista universitária em ensino de matemática e prática profissional de professores. Ela ministra cursos sobre tópicos relacionados à avaliação e gestão da aula de matemática para o ensino fundamental. É professora de futuros professores na área de Didática da Matemática e Metodologia de Pesquisa em Educação Matemática e História da Educação Matemática. Atualmente, está concluindo seu doutorado em Epistemologia e História da Ciência na Universidade de Tres de Febrero (Caseros, Buenos Aires, Argentina) e está escrevendo sua tese de doutorado.

E-mail: alejandraderiard@gmail.com

(ib) http://orcid.org/0000-0002-8201-3002 
Revista História da Educação (Online), 2020, v. 24: e99373 DOI: http://doi.org/10.1590/2236-3459/99373
e-ISSN: 2236-3459 http://seer.ufrgs.br/asphe

\footnotetext{
(c) (P)

Revista História da Educação - RHE

Associação Sul-Rio-Grandense de Pesquisadores em História da Educação - Asphe

Artigo de acesso aberto distribuído nos termos de licença Creative Commons.
} 